\title{
Radio over Fiber System Level Performance Analysis, Maintaining Signal Integrity
}

\author{
Rao Kashif, Oluwole John Famoriji, Fujiang Lin \\ Department of Electronic Engineering and Communication, University of Science and Technology, Hefei, China \\ Email:bila306@mail.ustc.edu.cn
}

How to cite this paper: Kashif, R., Famoriji, O.J. and Lin, F.J. (2017) Radio over Fiber System Level Performance Analysis, Maintaining Signal Integrity. Journal of Computer and Communications, 5, 1-8. https://doi.org/10.4236/jcc.2017.513001

Received: September 8, 2017

Accepted: November 6, 2017

Published: November 9, 2017

Copyright ( 92017 by authors and Scientific Research Publishing Inc. This work is licensed under the Creative Commons Attribution International License (CC BY 4.0).

http://creativecommons.org/licenses/by/4.0/ (c) (i) Open Access

\begin{abstract}
Radio over fiber is an integration of microwave and optical fiber technologies having numerous benefits. RoF technology can give a scope of advantages including the capacity for backing multiple radio services and standards. In order to maintain Signal Integrity in RoF system, it's indispensable to take all components of a system under rooted consideration. Due to the increasing number of RoF application, it's necessary to do some system level changes in designing RoF transceiver. In this paper we compared two popular modulation techniques NRZ (non return to zero) and RZ (return to zero) for better signal integrity in RoF system. Secondly we also did analysis of RoF transceiver with APD and PIN photo detectors and compared the performance on different distances. Hence the Q factor of PIN and APD photo diodes and comparison of NRZ and RZ modulation will be helpful for system designers who are working on better signal Integrity in Radio over fiber systems.
\end{abstract}

\section{Keywords}

RoF, PIN, APD, NRZ, RZ \& Photodiode

\section{Introduction}

With Radio over Fiber is just sending the radio signals over optical fiber. Easy maintenance, low power units, simple remote antennas, low attenuation loss, large bandwidth and reduced power consumption are few benefits of RoF link. RoF is an auspicious solution to accomplish the increasing demand of wireless and user bandwidth. In RoF system the only task of Base station is to convert optical signal into Radio signal. Figure 1(a) shows layout of simple bidirectional directly modulated RoF link. Modulation, multiplexing, coding are performed at Central Unit CU. The receiver consists of Photodiode to provide an RF power output proportional to the square of the input optical power. In recent 


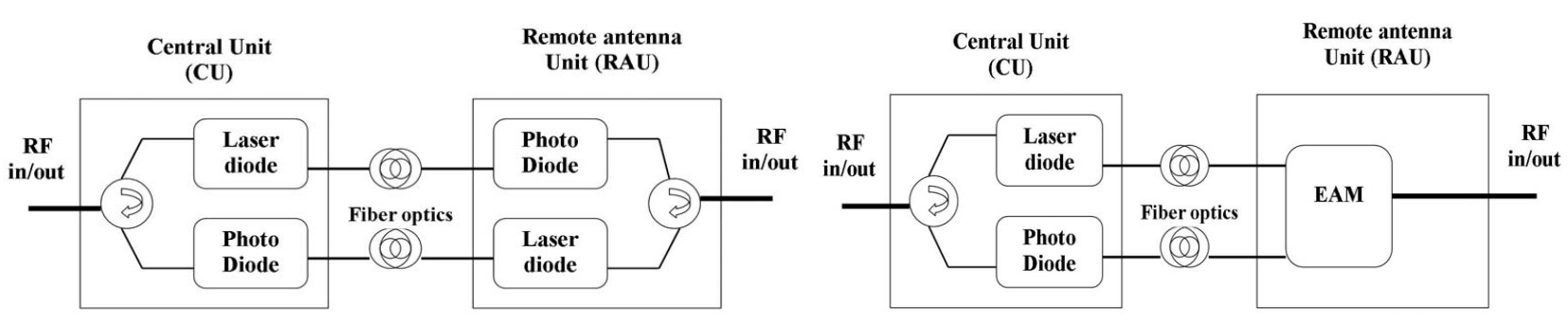

(a)

(b)

Figure 1. (a) Simple RoF link; (b) RAU with EAM.

advancement laser, photodiode and circulator are replaced with a single electronic device called electro absorption modulator [1] as shown in Figure 1(b). EAM acts as photodiode for downlink and modulator for uplink. No light source is needed at RAU which makes it much simpler and cheaper. In 2012 A. Kumar \& N. Agarwal worked on RoF integration microwave and optical communication access to broadband wireless communications [2]. Three methods implemented into RoF and compared with Attenuation, Scattering, BER and CNR.

Satellite Communication, mobile radio communication, broadband and wireless LAN are few application of RoF. Table 1 shows few advantages of RoF in mobile communication network [3] [4] [5] [6]. Laser diode non linearity is one of the major drawbacks of RoF which gives rise to inter modulation distortion and clipping noise. Signal Integrity is one of the biggest concerns for system designers [7] [8]. System level research and designing helps engineers to achieve their goals of signal Integrity in any network or systems.

\section{Modulation Techniques}

First step in optical system designing is to select right modulation technique. Modulation technique converts electrical signal into bit stream. We compared two well-known modulation NRZ (Non return to zero) and RZ (return to zero). Both techniques were analyzed with PRBS bit generator at $10 \mathrm{~GB}$ rate as shown in Figure 2.

The binary data should be encoded into electrical signal or optical waveform signal. NRZ is most commonly used binary data encoding. In NRZ bit period is the time given to transmitted bit where high amplitude is 1 and low amplitude is 0 and the sequence is called stream [9]. In RZ every bit will return to zero [10]. The pulse in NRZ have more energy than RZ one. Low cost and simple engineering makes NRZ most favorite. For optical communication system which is working on $10 \mathrm{G}$ or above the $\mathrm{RZ}$ increases transmit distance. In optical domain an extra Mach-Zehnder modulator is used, which is an expensive solution, But in electrical a high speed OR gate can be used as a cost efficient solution [11]. $\mathrm{NRZ}$ is not self-clocking so additional techniques should be used to avoid bit slip [11]. Synchronization in NRZ is handled via square wave signal known as bit clock [9].

In NRZ modulation the whole bit slot is occupied by 1 bit and no drop between two bits Figure 3. While in RZ modulation the amplitude return to zero 
Table 1. Advantages of Radio over Fiber.

\begin{tabular}{cc}
\hline No. & Advantages \\
\hline 1 & Good Coverage \\
2 & Reduction in number of handover \\
3 & Low maintenance cost \\
4 & Low fiber attenuation \\
5 & High Signal Quality \\
6 & Less System Design Cost \\
\hline
\end{tabular}

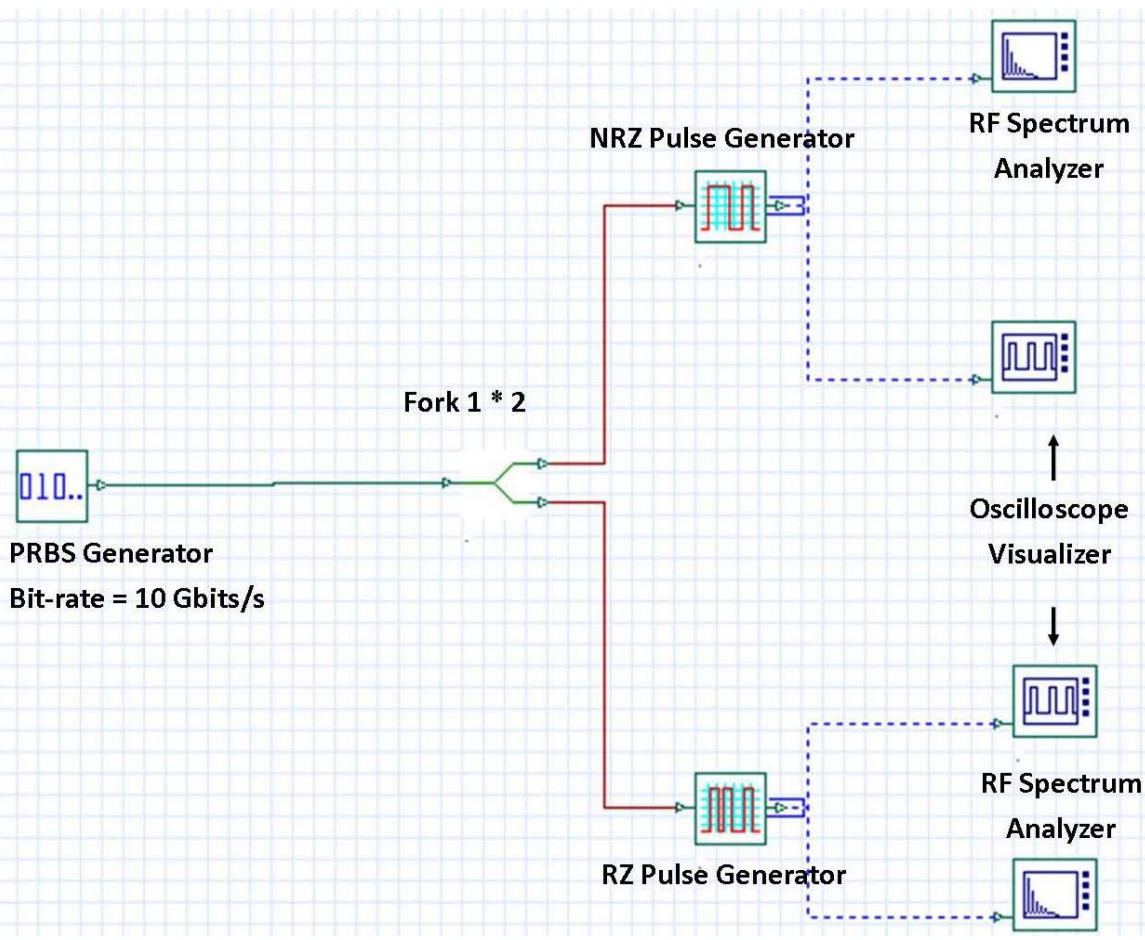

Figure 2. NRZ \& RZ modulation.

between each bit. NRZ took half the bandwidth as shown in Figure 4. So it's more data efficient. Finally eye diagram on receiver side of both NRZ \& RZ are shown in Figure 5.

\section{Radio over Fiber System Design Analysis}

Now a day's RoF applications are expanding and after significant contribution in telecommunication it's also benefiting the short range and LAN networks. While working on different scenarios for maintaining signal integrity in RoF network it's also mandatory to check the system design techniques and select right component for transceiver designed for specific networks or scenarios. Figure 6 shows a basic RoF system designed in Optic System 14.0. On receiver side two types of photo detectors PIN \& APD are used to compare the performance and select right photo detector for RoF receiver. 

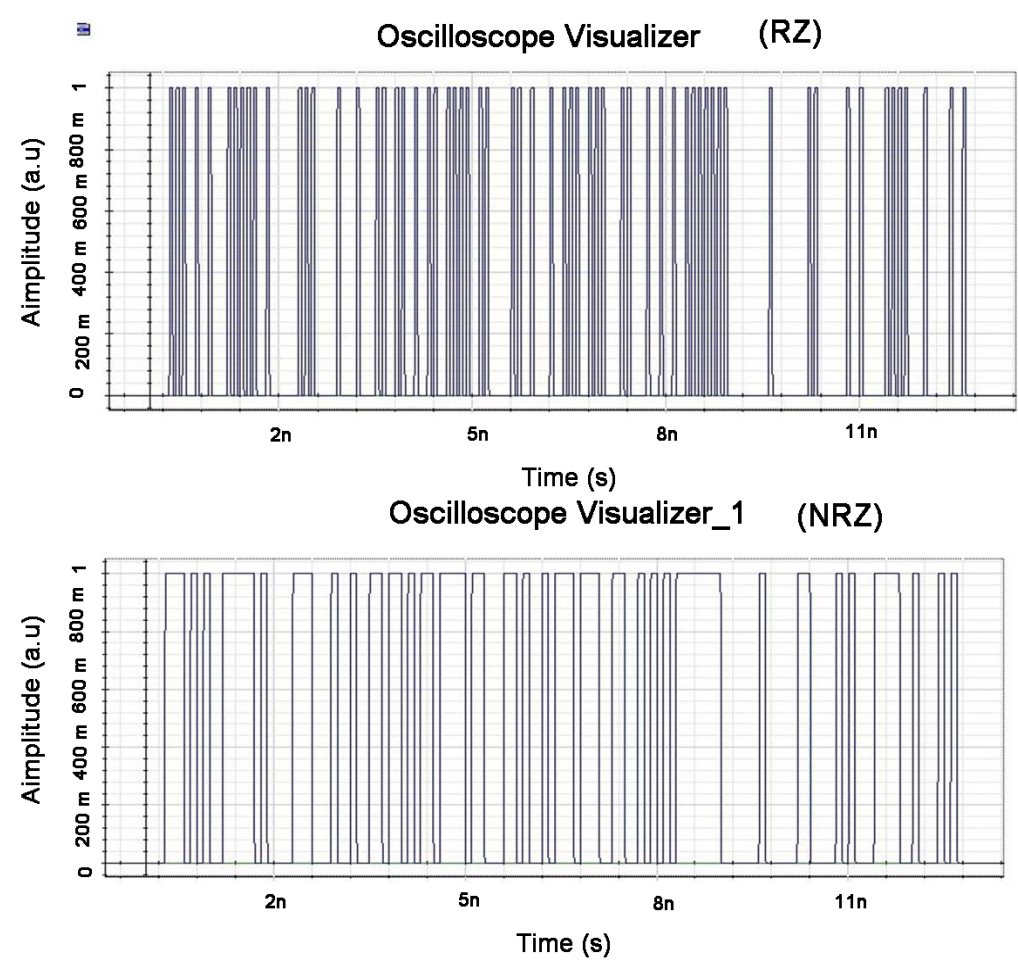

Figure 3. NRZ \& RZ oscilloscope analysis.

RF Spectrum Analyzer (RZ)

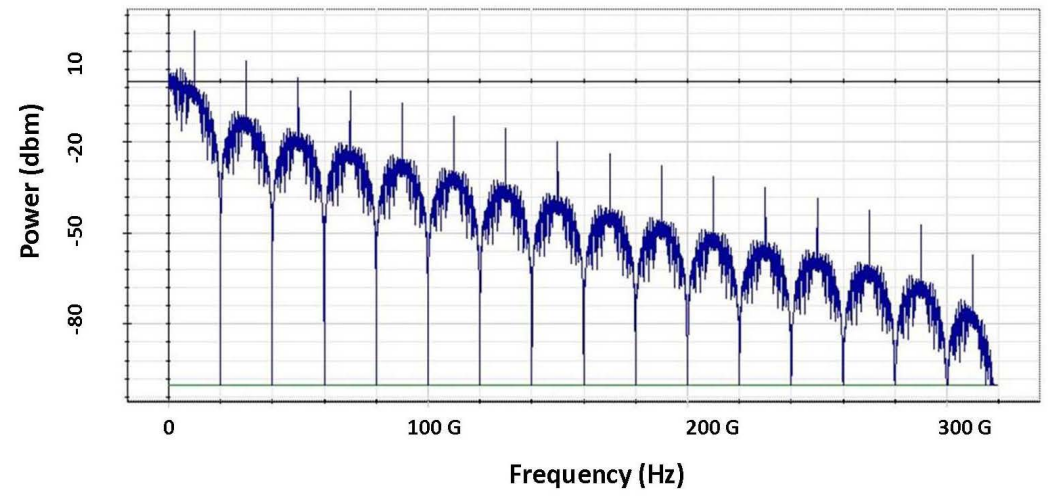

RF Spectrum Analyzer (NRZ)

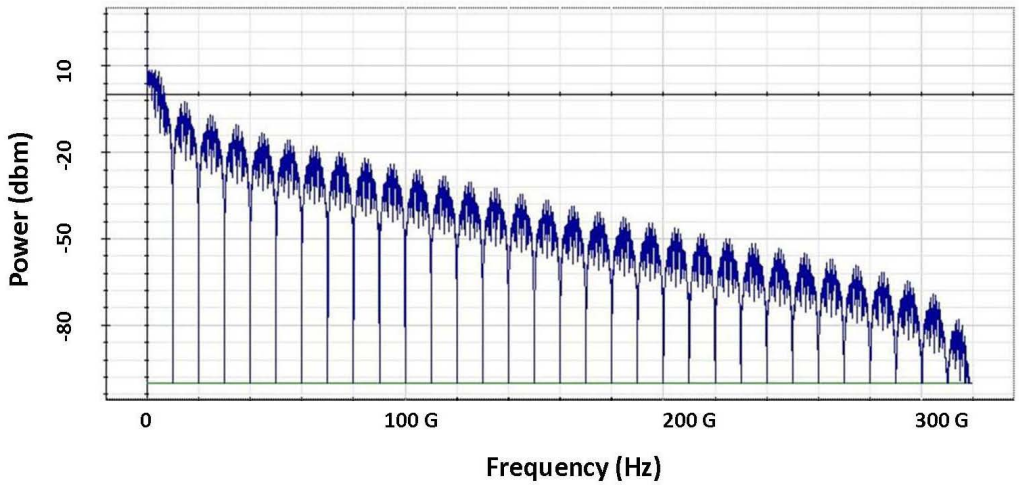

Figure 4. Rf spectrum Analysis. Finally eye diagram on receiver side of both NRZ \& RZ are shown in Figure 5. 


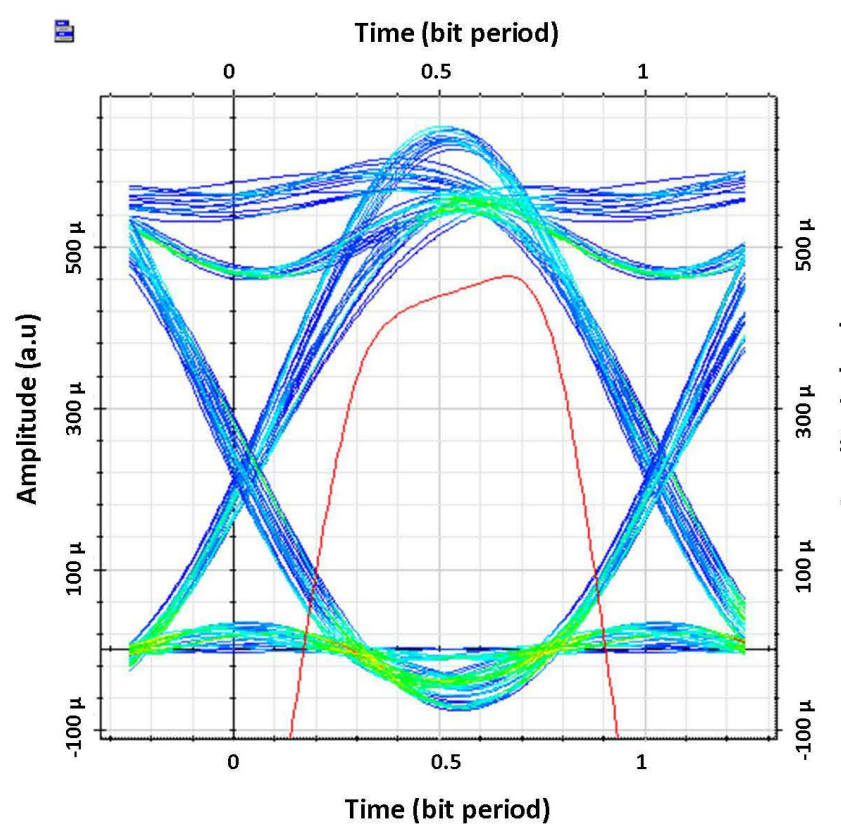

(NRZ)

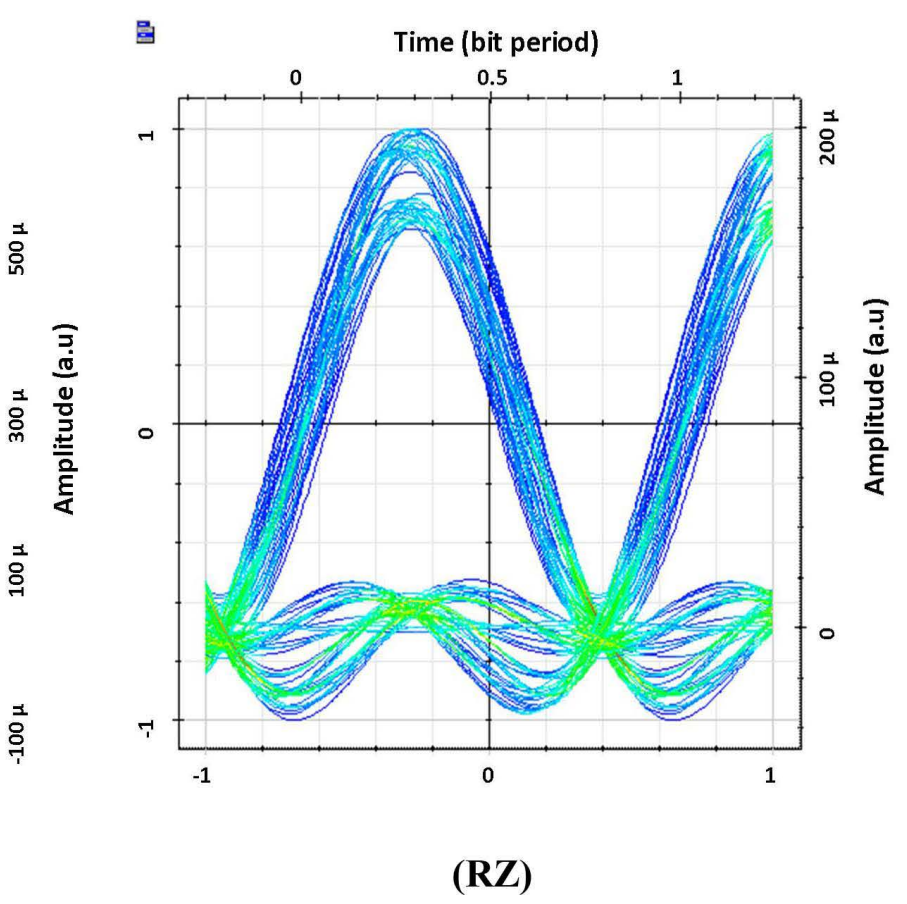

Figure 5. NRZ \& RZ eye diagram.

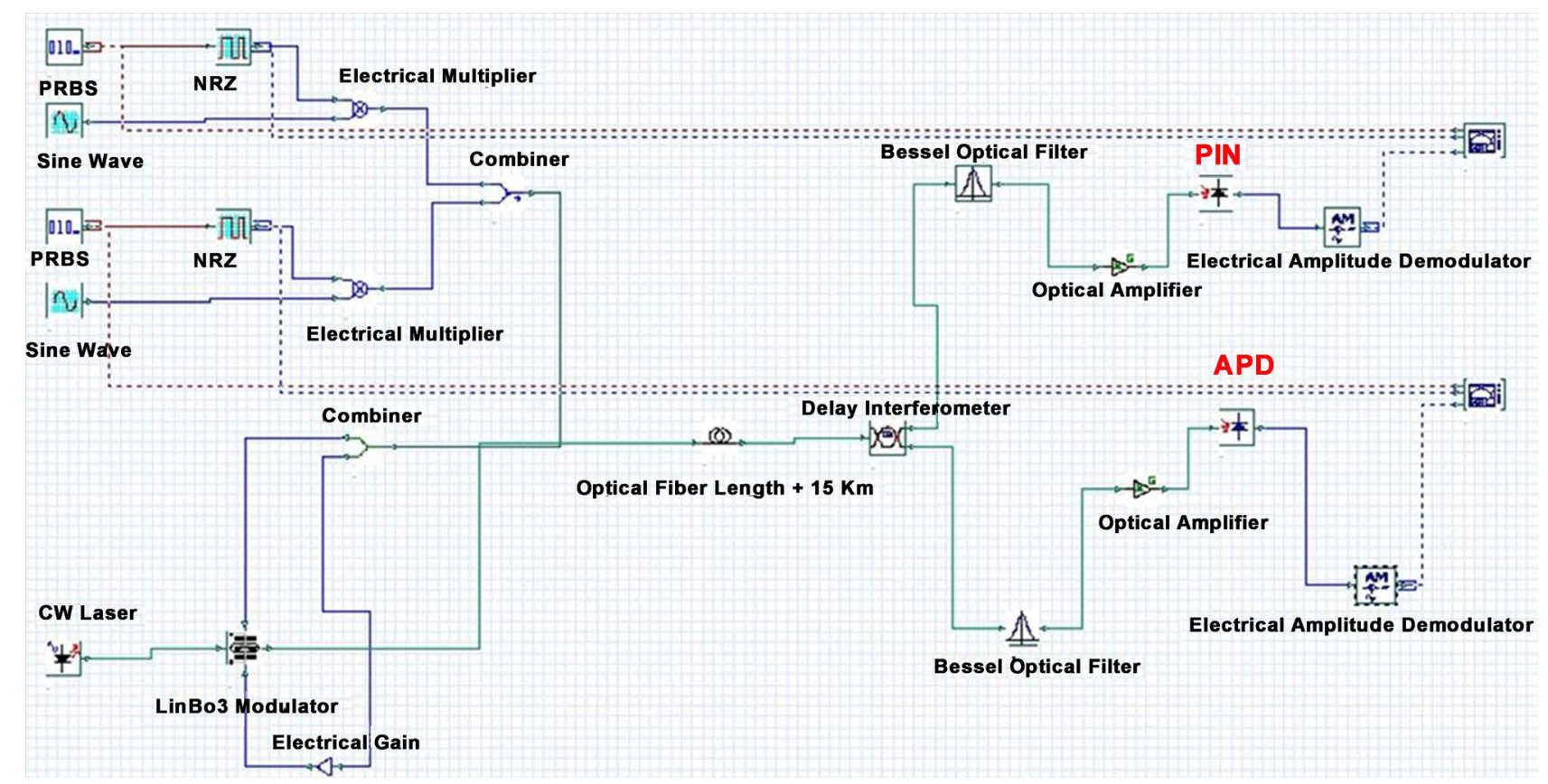

Figure 6. RoF system design with PIN \& APD Photo detector.

RoF is not all the time use for WAN but it also have applications in LAN. So keeping this in mind we tested the system with 5, 10, \& 15 Kilometers with both PIN \& APD photo detectors on receiver side. Performance of PIN photo detector was much better than APD at $5 \mathrm{Km}$ network, but as we start increasing the network area the APD photo detector show increase in Q-factor and on both 10 and $15 \mathrm{Km}$ the $\mathrm{q}$ factor of APD keeps increasing while reverse in case of PIN 


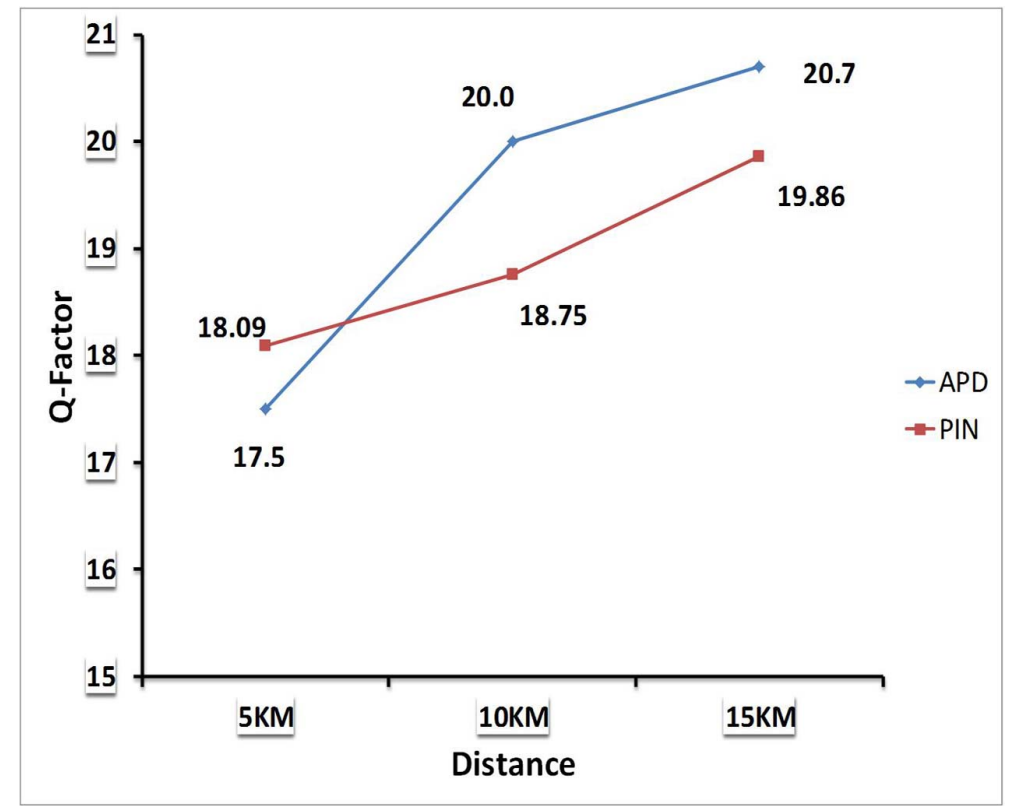

Figure 7. PIN \& APD Q-factor graph.

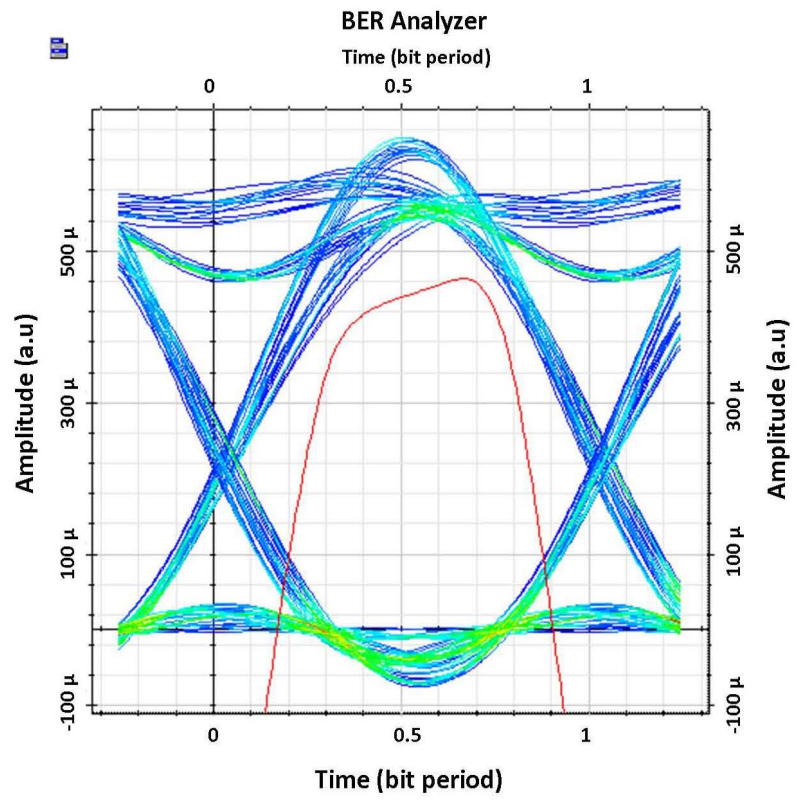

(APD) Photodiode

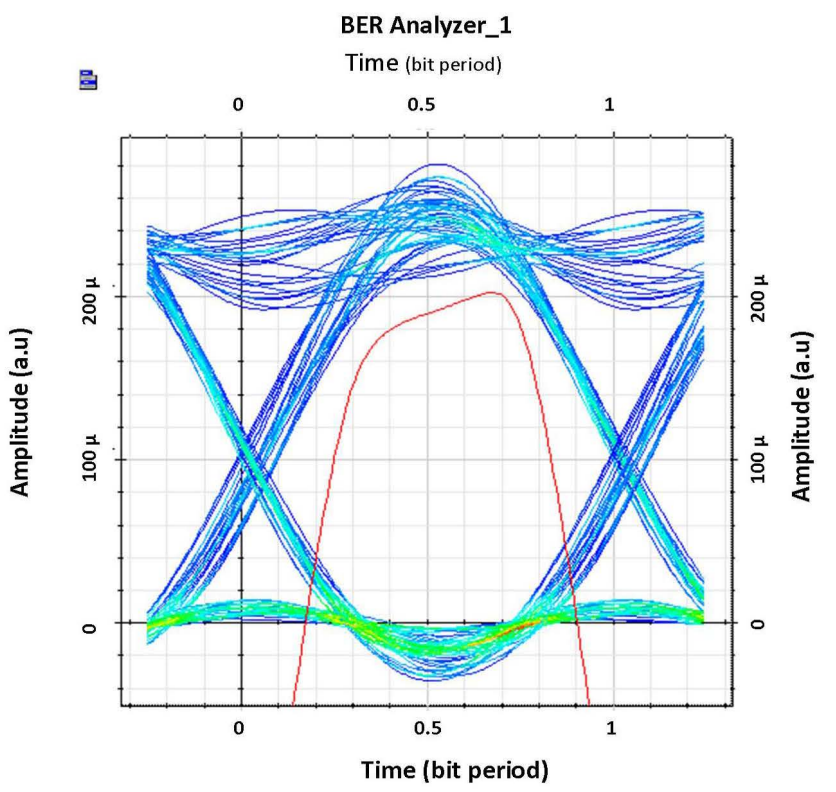

(PIN) Photodiode

Figure 8. APD and PIN photodiode Eye diagrams.

photo detector. Figure 7 shows the comparison of both photo detectors on 5, 10 and $15 \mathrm{Km}$ network. Figure 8 shows eye diagram of PIN and APD photo detector at 15 Kilometers.

As photo detector is one of most important component of RoF system so selecting a right photo detector can play great role in maintain signal integrity in system. From the above results we can see that transceivers with PIN photo detectors are suitable for LAN or campus communication RoF network as it shows 
better result on small distance communication while for WAN APD photo detector can be good candidate. The wide intrinsic region makes the PIN diode an inferior rectifier but make the PIN diode suitable for attenuators, fast switches, and high voltage power electronic applications. An avalanche photodiode (APD) is highly sensitive semiconductor device. Optical receiver with APD generally provides higher SNR for same optical power.

\section{Conclusion}

Two well-known modulation techniques NRZ and RZ were analyzed and NRZ (non-return to zero) was found more data efficient due to less bandwidth usage than RZ modulation. Secondly APD and PIN photo detectors were simulated in RoF system. From simulation results performed with different network lengths, it was cleared that APD is the best candidate for maintaining signal integrity in long networks. Selecting right modulation technique and Photo detector can improve the Integrity of Radio over Fiber system. Still work can be done on other components and improve system performance.

\section{Acknowledgements}

This research is supported by CAS-TWAS. We are thankful to all teacher and fellow colleague of MESIC for their contribution.

\section{References}

[1] Lach, E., et al. (2005) Application of Electro Absorption Modulators for High-Speed Transmission Systems. Journal of Optical and Fiber Communications Reports, 2, 140-170. https://doi.org/10.1007/s10297-005-0032-6

[2] Karthikeyan, R. and Prakasam, S. (2013) A Survey on Radio over Fiber (RoF) for Wireless Broadband Access Technologies. International Journal of Computer Applications, 64, No.12. https://doi.org/10.5120/10686-5587

[3] Al-Raweshidy, H. (2002) Radio over Fiber Technology for Next Generation. Artech House Publishers, Norwood.

[4] Kashif, R. and Lin, F.J. (2015) Signal Integrity Problems in Electronic Designing. Proceeding of. Asia-Pacific Microwave Conference (APMC), Nanjing, 6-9 December 2015. https://doi.org/10.1109/APMC.2015.7412979

[5] Way, W.I. (1993) Optical Fiber-Based Microcellular System: An Overview. IEICE Transactions on Communications, E76-B, 10911-11102.

[6] Kajiya, S., Ksukamoto, K. and Komaki, S. (1996) Proposal of Fiber-Optic Radio Highway Networks Using CDMA Method. IEICE Transactions on Electronics, E79-C, 496-500.

[7] Liu, J., et al. (2008) Waveguide-Integrated, Ultralow-Energy GeSi Electro-Absorption Modulators. Nature Photonics, 2, 433-437. https://doi.org/10.1038/nphoton.2008.99

[8] Fan, J., Ye, X.N., Kim, J., Archameault, B. and Orlandi, A. (2010) Signal Integrity Design for High-Speed Digital Circuits: Progress and Directions. IEEE Transaction on Electromagnetic Compatibility, 52, 392-400. https://doi.org/10.1109/TEMC.2010.2045381

[9] Maxin Integrated (2001) NRZ Bandwidth-HF Cutoff vs SNR. Application Note 870 
(HFAN-09.0.0). http://www.maximintegrated.com/en/app-notes/index.mvp/id/870

[10] Liang, H., Li, W., Linze, N. and Zhang, X. (2010) Comparison of Return-to-Zero and Non-Return-to-Zero Coded Pulses for BOTDA. The 9 th International Conference on Optical Communication and Networks (ICOCN 2010), Nanjing, 24-27 October 2010, 31-35. https://doi.org/10.1049/cp.2010.1146

[11] Schwanke, B. and Nellis, K. (2009) NRZ-to-RZ Data Conversion Using High Speed OR/AND. Electronic Product.

https://www.electronicproducts.com/Analog_Mixed_Signal_ICs/Communications_ Interface/NRZ-to-RZ_data_conversion_using_high-speed_OR_AND.aspx 